\title{
ANALYSIS OF JOVIAN LOW-FREQUENCY RADIO EMISSIONS BASED ON STEREOSCOPIC OBSERVATIONS WITH JUNO AND EARTH-BASED RADIO TELESCOPES
}

\author{
M. Imai*, W. S. Kurth*, G. B. Hospodarsky*, D. A. Gurnett*, \\ S. J. Bolton ${ }^{\dagger}$, J. E. P. Connerney ${ }^{\ddagger}$, S. M. Levin ${ }^{\S}$,

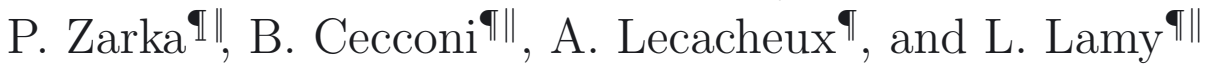

\begin{abstract}
Observations of Jupiter's low-frequency radio emissions were made using the radio and plasma wave instrument (Waves) onboard the Juno spacecraft in polar orbit around Jupiter since July 5, 2016. Waves is designed to monitor the electric fields of waves from $50 \mathrm{~Hz}$ to $41 \mathrm{MHz}$ with an electric dipole antenna and the magnetic fields of waves from $50 \mathrm{~Hz}$ to $20 \mathrm{kHz}$ with a magnetic search coil sensor. The Juno spacecraft rotates with a period of $30 \mathrm{~s}$, which modulates the spectral intensity sensed with the dipole antenna. We report early results of the Juno Waves investigation on (1) the direction-finding measurements of broadband kilometric (bKOM) radiation just before Juno's first perijove, (2) one concurrent decametric (DAM) radiation from the Juno spacecraft near Jupiter and the Nançay Decameter Array in France during Juno's interplanetary cruise, and (3) the statistical properties of Jovian DAM radio occurrence probability at $16 \mathrm{MHz}$ obtained from Juno data of June 21 to December 10, 2016, then compared with the previous statistical results of the Cassini and Voyager observations during their Jupiter flybys.
\end{abstract}

\section{Introduction}

Jupiter is the dominant auroral radio source in our solar system, producing Jovian lowfrequency radio emissions in a broad frequency range of $10 \mathrm{kHz}$ to $40 \mathrm{MHz}$ from both

\footnotetext{
* Department of Physics and Astronomy, University of Iowa, Iowa City, Iowa, USA

$\dagger$ Space Science Department, Southwest Research Institute, San Antonio, Texas, USA

¥ NASA Goddard Space Flight Center, Greenbelt, Maryland, USA

$\S$ Jet Propulsion Laboratory, California Institute of Technology, Pasadena, California, USA

${ }^{I}$ LESIA, Observatoire de Paris, CNRS, PSL, UPMC/SU, UPD, Place J. Janssen, Meudon, France

" Station de Radioastronomie de Nançay, Obs. Paris, CNRS, PSL, Univ. Orléans, Nançay, France
} 
north and south polar regions of the planet. According to the spectral morphology, three types of auroral radio emissions are known as broadband kilometric (bKOM) radiation from $10 \mathrm{kHz}$ to $1 \mathrm{MHz}$, hectometric $(\mathrm{HOM})$ radiation from $300 \mathrm{kHz}$ to $10 \mathrm{MHz}$, and decametric (DAM) radiation from a few to $40 \mathrm{MHz}$ [cf. Carr et al., 1983; Zarka, 1998; Clarke et al., 2004; Imai et al., 2011, and references therein]. These emissions have common features: (1) sporadic nonthermal radio bursts, (2) highly circular or elliptical polarization, (3) emission frequency very close to the local gyrofrequency at the source via the electron cyclotron maser instability (CMI) [cf. Treumann, 2006], and (4) anisotropic emission beam from a radio source along a magnetic flux tube.

During Juno's interplanetary cruise prior to Jupiter orbit insertion on July 5, 2016, the first Jovian radio observations were recorded with one of Juno's instruments called Waves [Kurth et al., 2017a] for bKOM in March, and for HOM and DAM radiation in May 2016. Since then, a new opportunity is provided to capture the entire Jovian low-frequency radio spectrum from one single spacecraft long after the Voyager spacecraft in 1979 [Warwick et al., 1977; Scarf and Gurnett, 1977]. Juno entered a polar orbit of Jupiter on July 5, 2016, with a perijove distance of 1.06 Jovian radii $\left(R_{J}, 71492 \mathrm{~km}\right)$. This initial closest approach to Jupiter is referred to as Perijove 0 (PJ0), and the subsequent orbits are enumerated sequentially. The first scientific perijove (PJ1) observations were made by Juno from pole to pole, capturing complex auroral activities in a broad range of electromagnetic wavelengths [Kurth et al., 2017c]. Waves is capable of recording the electric fields of waves from $50 \mathrm{~Hz}$ to $41 \mathrm{MHz}$ with one electric dipole antenna and the magnetic fields of waves from $50 \mathrm{~Hz}$ to $20 \mathrm{kHz}$ with one magnetic search coil sensor [Hospodarsky, 2016]. Together with the two sensors, Waves has three on-board receivers consisting of the Low Frequency Receiver (LFR) and two redundant High Frequency Receivers (HFR), operating in five different frequency bands for the measurements of magnetic and electric fields of waves. In this study, we utilize three frequency bands, the higher and lower frequency bands of the LFR (LFRH and LFRL) and the higher frequency band of the HFR (HFRH). The LFRL and LFRH, respectively, comprise 43 approximately logarithmically-spaced frequency channels from $48.83 \mathrm{~Hz}$ to $19.97 \mathrm{kHz}$, and 18 approximately logarithmicallyspaced frequency channels from $19.96 \mathrm{kHz}$ to $141.5 \mathrm{kHz}$. The HFRH consists of 38 linearly spaced channels from 3.5 to $40.5 \mathrm{MHz}$ with a noise bandwidth of $666 \mathrm{kHz}$. The noise bandwidth is the width of an ideal square filter with the same response as the HFRH channel. Depending upon the mode operation of the instrument, the temporal resolution is selectable at $1,2,10$, or 30 seconds for all of three frequency bands.

In this paper, we focus on three phenomena of the Jovian bKOM and DAM radio emissions analyzed during Juno's approach and initial orbits of Jupiter. First, we perform the direction-finding (DF) measurements for bKOM just before PJ1. Second, we show a stereoscopic observation of Jovian DAM emission with Juno and the Nançay Decameter Array (NDA) in France during Juno's interplanetary phase. Finally, we investigate statistical properties of the Jovian radio emission occurrence probability at $16 \mathrm{MHz}$ using Juno data from June 21 to December 10, 2016, and compare it with the Cassini and Voyager statistical results at about $16 \mathrm{MHz}$. The vantage point of Juno in latitude and the wide frequency coverage of Waves from $50 \mathrm{~Hz}$ to $41 \mathrm{MHz}$ lead to a better understanding of the bKOM radio source locations from Juno direction finding, the DAM beaming properties using the coordinated DAM observations with Juno and ground-based radio observato- 
(a) Juno/Waves/LFRL\&LFRH 2016-08-26 22:15 through 2016-08-27 00:15
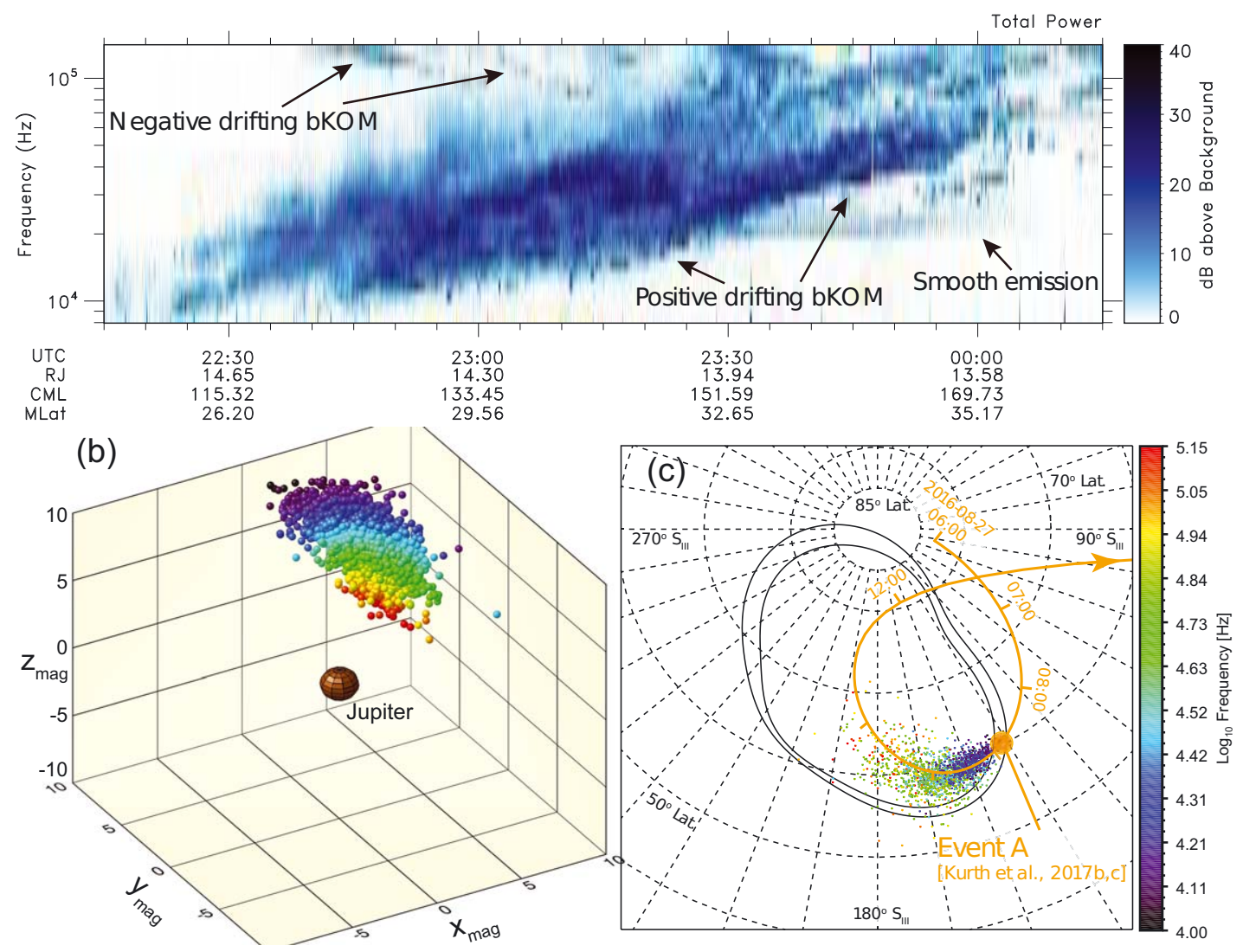

Figure 1: (a) Jovian dynamic spectrum in a frequency range of 8.94 to $141.5 \mathrm{kHz}$, (b) 3-D positions of bKOM radio sources derived from the Juno Waves instrument in Jovian magnetic coordinates, and (c) these radio sources mapped along magnetic field lines onto the Jovian northern atmosphere at the 1-bar level. The color code represents frequency from 10 to $141.5 \mathrm{kHz}$ on a logarithmic scale with a total of 24 channels as the color changes from black to red. In (c), the statistical UV auroral oval taken from the Hubble Space Telescope [Bonfond et al., 2012] is depicted by the black solid curves and the Juno footprints (i.e., points where the magnetic fields of Juno positions are traced onto the Jovian atmosphere) are plotted as orange curves with 1 hour tick marks. In addition, the orange circle shows the Juno footprint during a near-source crossing classified as Event A in Kurth et al. [2017b,c].

ries, and the statistical DAM morphology at $16 \mathrm{MHz}$ compared with Cassini and Voyager observations.

\section{Juno DF analysis for bKOM}

Just prior to PJ1 on August 27, 2016, Juno detected strong radiation in the frequency range of 8.94 to $141.5 \mathrm{kHz}$ from 22:15 on August 26 to 00:15 on August 27, 2016, as shown in Figure 1a. There are primarily positive drifting components, a few negative drifting components, and a smooth component. The former two are types of bKOM, while the latter is escaping continuum radiation. Here, we are only interested in the positive drifting bKOM components. By means of the modulated spectral intensity from 
the dipole antenna aboard the Juno spinning spacecraft [Sampl et al., 2016], we perform a two-dimensional determination of the direction of arrival of the positive drifting bKOM component. For the case of purely circular polarized emissions like bKOM [e.g., Ladreiter et al., 1994], the received spectral density of the wave electric field $W$ can be described, according the antenna theory [Lecacheux, 1978; Cecconi, 2007], as

$$
W=W_{0}\left[\left(1-\frac{m^{2}}{2}\right)-\frac{m^{2}}{2} \cos \left[2\left(\omega t-\phi_{\mathrm{SC}}\right)\right]\right]
$$

where $W_{0}$ is the true wave amplitude and $\omega t$ is the rotation angle of spacecraft, and modulation index $m$ is $\sin \theta_{\mathrm{SC}}$ if a radio source is expressed as a point source. $\theta_{\mathrm{SC}}$ and $\phi_{\mathrm{SC}}$ are the zenith and azimuth angles, respectively, of the wave $\mathbf{k}$ vector in the Juno spacecraft frame. These angles can be determined using a least-squares fit of the observed spectral density for each frequency channel versus rotation phase, and then translated into Jovian magnetic coordinates using the VIP4 dipole [Connerney et al., 1998]. Furthermore, assuming that the waves are propagating in the right-hand extraordinary ( $\mathrm{R}-\mathrm{X})$ mode, we derive a radio source location by finding an intersection of the derived wave $\mathbf{k}$ vector and the R-X cutoff surface via the straight-line propagation. The R-X cutoff frequency is defined as $f_{R X}=\sqrt{\left(f_{g} / 2\right)^{2}+f_{p}^{2}}+f_{g} / 2$, where $f_{p}$ and $f_{g}$ are the electron plasma frequency and gyrofrequency, respectively. The Jovian magnetic field and plasma density models are the same as in Imai et al. [2015], but excluding a magnetic flux shell density model.

Figure $1 \mathrm{~b}$ shows the three-dimensional (3-D) positions of bKOM radio sources computed from the described direction-finding method. The color code indicates the frequency from 10 to $141.5 \mathrm{kHz}$ with a total of $24 \log$-spaced channels. As the emission frequency increases, the positions of bKOM radio sources tend to be closer to Jupiter in the northern hemisphere. In Figure 1c, these radio source locations are mapped along magnetic field lines onto the Jovian northern atmosphere, superimposed with the statistical UV auroral oval observed by the Hubble Space Telescope [Bonfond et al., 2012] and the footprints of Juno during a near in-situ crossing of a source detected by Juno (Event A in Kurth et al. [2017b,c]). Our bKOM radio footprints are close to the inner edge of the auroral oval, in good agreement with the Juno footprints during the near-source crossing [Kurth et al., 2017b,c]. The Ulysses DF study done by Ladreiter et al. [1994] showed that the southern bKOM radiation at 81 and $100 \mathrm{kHz}$ came from auroral regions. Imai et al. [2017a] further investigated the same bKOM event, finding that the M-shell (similar to L-shell but with a non-dipole field model) of these radio sources ranges from 50 to 60, compared to Mshell $=5-58$ [Ladreiter et al., 1994], and the mean cone half-angle extends from $40^{\circ}$ to $55^{\circ}$, consistent with that of $30^{\circ}$ to $80^{\circ}$ based on the Ulysses DF study [Ladreiter et al., 1994]. Therefore, our result provides additional evidence that northern bKOM radio sources are located along high latitude auroral magnetic field lines. For further detailed analysis of the radio source parameters and beaming properties of the bKOM radiation, see Imai et al. [2017a]. 


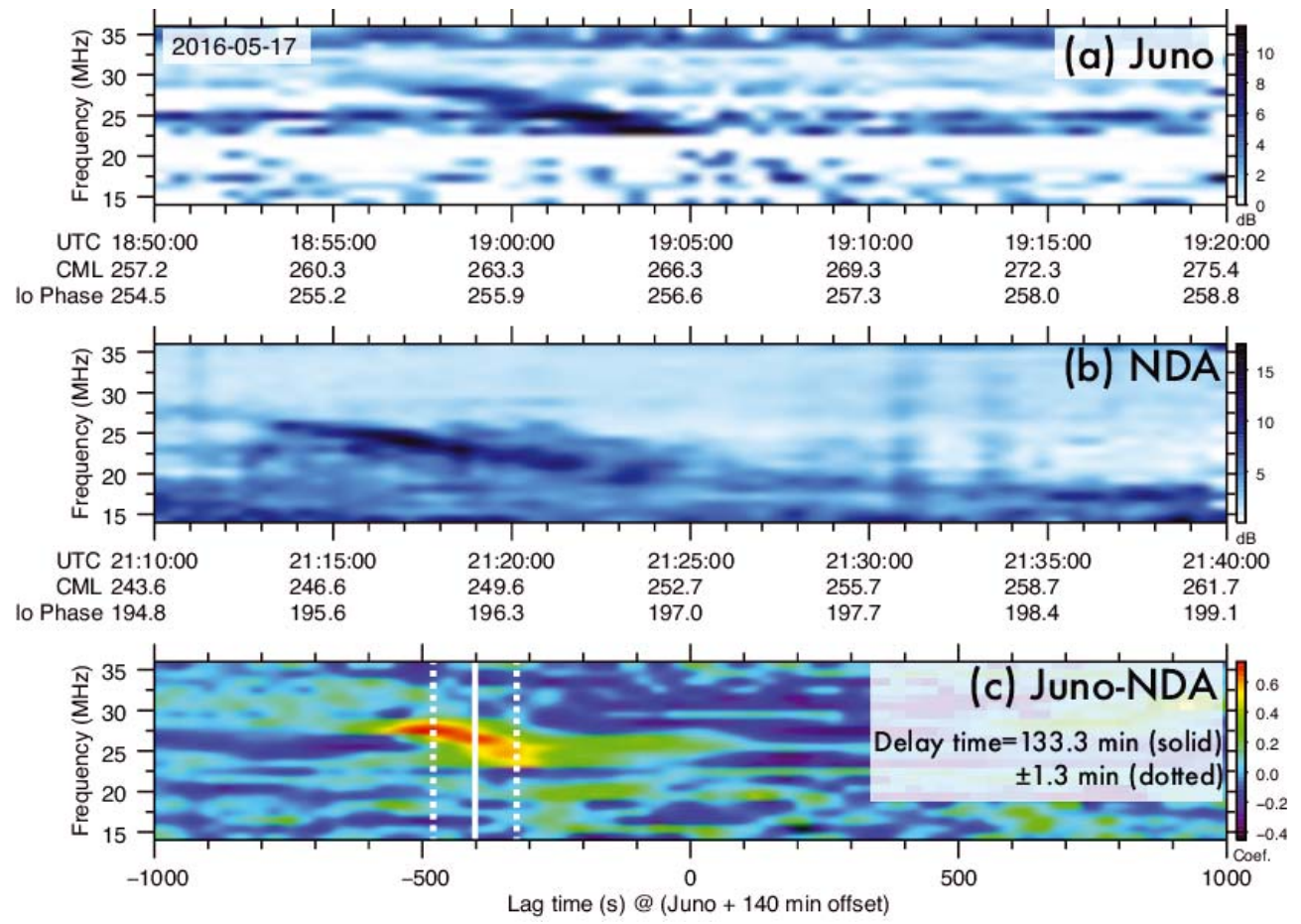

Figure 2: Jovian dynamic spectra from (a) Juno and (b) NDA, and (c) cross-correlation spectrum in a pair of Juno and NDA. Following the cross-correlation method by Imai et al. [2016], the Juno and NDA spectral data were averaged over a spectral resolution of $1 \mathrm{MHz}$ and a temporal resolution of $30 \mathrm{~s}$. The correlated discrete arc (CDA) is extracted from the radio frequency interference and hourly routine calibration signals for Juno and NDA. The difference in the starting time observed by Juno and NDA is $140 \mathrm{~min}$. The delay time is the lag time at the cross-correlation peak plus the offset time of $140 \mathrm{~min}$. The white color vertical solid line is the averaged delay time of $133.3 \mathrm{~min}(=-400 \mathrm{~s}+140 \mathrm{~min}$ ), while the white color vertical dotted lines are the averaged value plus and minus one standard deviation (133.3 $\mathrm{min} \pm 1.3 \mathrm{~min})$.

\section{Jovian DAM radiation as viewed from Juno and NDA}

Using the Juno spacecraft near Jupiter and ground-based radio telescopes at Earth, stereoscopic DAM observation is another tool with which to understand the CMI emission mechanism and its beaming properties. One of the powerful low-frequency radio telescopes at Earth is the NDA [Boischot et al., 1980; Lecacheux, 2000], located in Nançay, France, consisting of 72 right- and 72 left-hand conical spiral antennas. Jupiter observations are acquired with the NDA digital routine receiver in operation since 1990 [Lamy et al., 2017]; the power recorded in the RH and LH sub-arrays are recorded over a total of 400 linearly spaced channels between 10 and $40 \mathrm{MHz}$ with a noise bandwidth of $30 \mathrm{kHz}$. The routine receiver is a swept frequency receiver with a $87 \mathrm{~ms}$ integration time. The $\mathrm{RH}$ and $\mathrm{LH}$ signals are measured alternatively every $0.5 \mathrm{~s}$. Hence, the overlapping frequency range between Juno and the NDA covers the range from 10 to $40 \mathrm{MHz}$, most of the Jovian DAM spectrum.

We have identified one concurrent DAM emission captured from Juno and NDA on May 17, 2016, hereafter called the correlated discrete arc or CDA. The CDA recorded by NDA shows stronger RH polarized power than LH, suggesting that this emission should come 
from the Jovian northern pole region assuming R-X mode emission [Carr et al., 1983]. Accordingly, only RH signals are used in this study. The distance from Jupiter was $424 R_{J}$ with $9.7^{\circ}$ Jovicentric latitude for Juno and $1.06 \times 10^{4} R_{J}$ with $-1.5^{\circ}$ Jovicentric latitude for NDA. Of particular interest is the cause of the delay of the CDA between Juno and NDA. To do so, following the cross-correlation method used by Imai et al. [2016], we carried out two steps: resampling process and cross-correlation analysis. In the former step, because of the different temporal and spectral resolutions of data for Juno and NDA, we averaged the observed spectral density over a spectral resolution of $1 \mathrm{MHz}$, and then resampled the obtained values with a common fixed temporal resolution of $30 \mathrm{~s}$. These spectral data are shown in Figure 2a for Juno and Figure 2b for NDA. It is important to note that, because of the severe radio frequency interference and hourly routine calibration signals in the NDA dynamic spectrum, only 30-minutes of data when the CDA appears for Juno and NDA is extracted. By cross-correlating these spectral data, the cross-correlation spectrum is obtained in Figure 2c, where the mean $(\mu)$ and standard deviation $(\sigma)$ of the delay time are $133.3 \mathrm{~min}$ and $1.3 \mathrm{~min}$, respectively.

The delay time of the CDA at the two receiving stations can be accounted for by three contributions: (1) the propagation of the radio waves, (2) the location of the radio source, and (3) the geometry of the 3-D beaming structure. The first contribution is due to the light travel time difference between two distant stations determined to be $40.5 \mathrm{~min}$. The second contribution is related to the rotation of the beam sweeping from Juno to NDA. In the Jovian system, two possible bodies controlling the rotation of the beam are the orbital motion of Io at a period of $42.46 \mathrm{~h}$ and Jupiter's rotation at a period of $9.925 \mathrm{~h}$. Because the stereoscopic azimuth angle between Juno and Earth is $73.8^{\circ}$, the rotational time is $522.2 \mathrm{~min}$ for the orbital motion of Io and $122.0 \mathrm{~min}$ for Jupiter's System III rotation. The remaining delay after subtracting the light time delay is $92.8 \pm 1.3 \mathrm{~min}$, which is much smaller than the former rotational time and is close to but slightly different from the latter rotational time. To solve this problem, the third contribution from the cross-section of the beaming pattern as seen from two latitudinally different observers must be included. This comes from the fact that the radio beam is formed as a fixed thin hollow cone along an active magnetic flux tube, where a radio source is excited at a frequency approximately equal to the local gyrofrequency. Using the fixed hollow cone model, Imai et al. [2017b] successfully reproduced the spectral structure of the CDA observed by Juno and the NDA, where these radio sources, mapped along magnetic field lines at an M-shell $\geq 8$ onto the Jovian northern atmosphere, are localized within $173^{\circ} \pm 10^{\circ}$ System III longitude. Furthermore, the corresponding resonant electron energy is from 0.5 to $11 \mathrm{keV}$. Additional stereoscopic DAM observations with Juno and Earthbased radio telescopes will give a further insight into our understanding of the CMI radio source properties and the latitudinal beaming properties of Jovian DAM radiation [Imai et al., 2017b].

\section{Jovian radio occurrence probability at $16 \mathrm{MHz}$}

From a statistical point of view, the occurrence probability of radio emissions may be used for examining the morphology of Jupiter's DAM radio emissions. In determining 

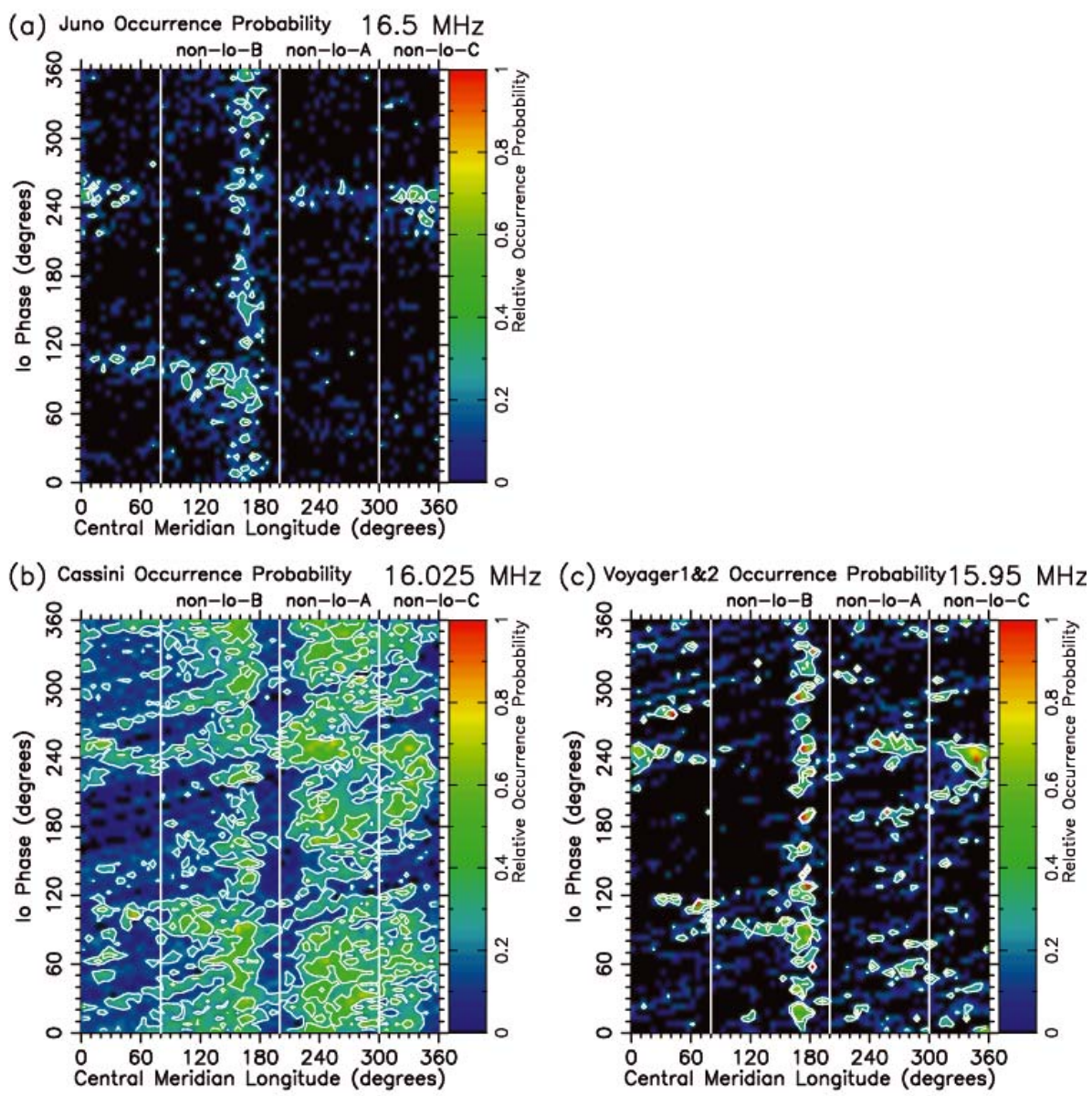

Figure 3: Occurrence probability maps at $16 \mathrm{MHz}$ for (a) Juno, (b) Cassini, and (c) combined Voyager 1 and 2 plotted as a function of System III CML and Io phase. The Cassini and Voyager occurrence probability maps are adapted from Imai et al. [2008; 2011]. The white contours correspond to occurrence probability levels of $20 \%$ and $40 \%$.

the occurrence probability of DAM radiation, we applied the rotation-based averaging method [e.g. Imai et al., 2008; 2011] to the Juno Waves data at $16.5 \mathrm{MHz}$ from June 21 to December 10, 2016, at distances ranging from 30 to $158 R_{J}$. We excluded observations inside of $30 R_{J}$ due to the rapid variation of spacecraft location. In the analyzed interval, Juno's latitude changes from $-21^{\circ}$ to $+15^{\circ}$, while its local time (LT) varies from $6.2 \mathrm{~h}$ to $5.4 \mathrm{~h}$. The data are normalized to the intensity at a distance of $1000 R_{J}$ over one Jupiter rotation. We determined a detection threshold defined as the mean intensity $(\mu)$ plus two standard deviations $(2 \sigma)$ of the mean. The occurrence probability is the number of detections above this threshold divided by the number of observations in $5^{\circ}$ bins of System III CML and Io phase.

In addition to the Juno occurrence probability of $16.5-\mathrm{MHz}$ DAM emission in Figure 3a, the Jovian radio occurrence probability around $16 \mathrm{MHz}$ is incorporated into Figure $3 \mathrm{~b}$ for Cassini data [Imai et al., 2008; 2011] and into Figure 3c for combined Voyager 1 and 2 data [Imai et al., 2011] using the same rotation-based averaging method but different detection thresholds. The occurrence probability is organized as a function of Io phase and 
Jovian CML. Comparing Juno, Cassini, and Voyager, there is a relatively high occurrence probability in a narrow range of Io phases at $80^{\circ}$ and at $250^{\circ}$, corresponding to the Iorelated emissions, independent of the different latitude ranges of the spacecraft. This implies that the Io-DAM beam extends broadly in latitude. More striking, the non-IoA/C occurrence probability between $200^{\circ}$ and $360^{\circ} \mathrm{CML}$ is dominant for Cassini but less prominent for Juno and Voyager. This may be influenced by the local time of the observations: Cassini (at $11 \mathrm{~h}$ and $21 \mathrm{~h} \mathrm{LT}$ ), Voyager 1 (at $10 \mathrm{~h}$ and $4 \mathrm{~h} \mathrm{LT}$ ), and Voyager 2 (at $10 \mathrm{~h}$ and $3 \mathrm{~h} \mathrm{LT}$ ). This effect is referred to as the local time effect [Alexander et al., 1981; Leblanc, 1981] and the physical mechanism is possibly a change of electron density in the Jovian ionosphere and a change of electron precipitation along an active magnetic flux tube. Comparing Jupiter's DAM occurrence before and after the Voyager encounter to Jupiter, Alexander et al. [1981] and Leblanc [1981] pointed out the non-Io-DAM radio emissions (including all of non-Io-A/B/C components) are influenced by this effect. In contrast, the non-Io-B occurrence probability between $80^{\circ}$ and $200^{\circ} \mathrm{CML}$ is observed for all spacecraft. Therefore, the non-Io-B emission is not controlled by the local time effect, but rather Jovian System III rotation. By incorporating more spectral data in a wide frequency of 3.5 to $40.5 \mathrm{MHz}$, this non-Io-B structure observed by Juno was further analyzed by Imai et al. [2017c], who pointed out the latitudinal and spectral dependence of the appearance of the non-Io-B occurrence probability.

\section{Summary}

We have investigated the Jovian broadband kilometric (bKOM) radiation and decametric (DAM) radiation during the interplanetary cruise of Juno spacecraft and after Jupiter orbit insertion of Juno by analyzing three phenomena, which are summarized as follows.

- By means of Juno directing finding for the bKOM radiation recorded from 22:15 on August 26 to 00:15 on August 27, 2016, the northern bKOM radio sources at 10 to $141.5 \mathrm{kHz}$ are localized at high latitudes on auroral magnetic field lines. The bKOM radio source locations mapped along magnetic fields onto Jupiter's northern atmosphere are localized near the inner edge of the empirical main oval.

- A concurrent DAM arc was observed with a latitudinal separation of $11^{\circ}$ from the Juno spacecraft near Jupiter and the Nançay Decameter Array (NDA) on May 17, 2016. Using a cross-correlation analysis, the delay time from Juno to NDA is $133.3 \pm 1.3 \mathrm{~min}$, which can be considered to be a combination of the light travel time, rotational time of a radio source fixed in Jovian System III longitude, and the shift in time due to the cross-section of the beaming pattern at different latitudes.

- Using the Juno data from June 21 to December 10, 2016, the Jovian radio occurrence probability at $16 \mathrm{MHz}$ was produced, subsequently compared with the previous statistical results of Cassini and Voyager data near $16 \mathrm{MHz}$. The non-IoB occurrence probability is clearly exhibited for all spacecraft regardless of their local times and latitudes. However, the local time effect dominates the non-Io-A occurrence probability for Cassini but less for Juno and Voyager. 
Juno has three advantageous methods to determine the radio source locations and the beaming properties for the Jovian low-frequency radio emissions. First, identifying emission frequency close to the local gyrofrequency and in-situ particle measurements through Juno's perijove surveys from pole to pole, the locations and generation mechanism of auroral radio sources can be determined. Juno's proximity to Jupiter is a prime advantage. During the Juno perijove 1 exploration, Kurth et al. [2017b,c] found five or more radio source regions with Juno and Louarn et al. [2017] pointed out that the loss cone driven CMI theory is able to reproduce the observed HOM radio emission. Second, the spinmodulated spectral density recorded with Juno Waves yields the positions of Jovian radio sources. This was done by Imai et al. [2017a] for Jovian low-frequency radiation during Juno's first perijove. Finally, on the basis of the Jovian radio beaming model, performing stereoscopic radio observations with Juno and an Earth-based radio telescope [Imai et al., 2017b] or investigating the statistical characteristics of Jovian radio occurrence [Imai et al., 2017c] boosts our knowledge of the emission mechanism and beaming properties. Because the three individual methods are self-consistent and complement each other, Juno observations are useful for determining the Jovian radio beam parameters and radio source locations, which can be traced along magnetic field lines onto Jupiter's atmosphere and further compared with the UV and infrared auroral emissions. More low-frequency radio observations by Juno in concert with Earth-based radio telescope observations are anticipated through the on-going Juno mission.

Acknowledgments. The research at the University of Iowa was supported by NASA through Contract 699041X with the Southwest Research Institute, and the Theodore Dunham, Jr. grant from the Fund for Astrophysical Research. The NDA is hosted by the Nançay Radio Observatory / Unité Scientifique de Nançay of the Observatoire de Paris (USR 704-CNRS, supported by Université d'Orléans, OSUC, and Région Centre in France). The responsible Editor thanks Mykhaylo Panchenko and one anonymous reviewer for their help in evaluating this paper.

\section{References}

Alexander, J.K., T. D. Carr, J. R. Thieman, J. J. Schauble, and A. C. Riddle, Synoptic observations of Jupiter's radio emissions: Average statistical properties observed by Voyager, J. Geophys. Res., 86, 8529-8545, 1981.

Bonfond, B., D. Grodent, J.-C. Gérard, T. Stallard, J. T. Clarke, M. Yoneda, A. Radioti, and J. Gustin, Auroral evidence of Io's control over the magnetosphere of Jupiter, Geophys. Res. Lett., 39, L01105, 2012.

Boischot, A., C. Rosolen, M. G. Aubier, G. Daigne, F. Genova, Y. Leblanc, A. Lecacheux, J. de la Noë, and B. M. Pedersen, A new high gain, broadband steerable, array to study Jovian decametric emission, Icarus, 43, 399-407, 1980.

Carr, T. D., M.D. Desch, and J.K. Alexander, Phenomenology of magnetospheric radio emissions, in Physics of the Jovian Magnetosphere, edited by A. J. Dessler, Cambridge University Press, New York, USA, 226-284, 1983. 
Cecconi, B., Influence of an extended source on goniopolarimetry (or direction finding) with Cassini and Solar TErrestrial RElations Observatory radio receivers, Radio Sci., 42, RS2003, 2007.

Clarke, J. T., D. Grodent, S. W. H. Cowley, E. J. Bunce, P. Zarka, J. E. P. Connerney, and T. Satoh, Jupiter's aurora, in Jupiter: the Planet, Satellites, and Magnetosphere, edited by F. Bagenal, W. McKinnon, and T. Dowling, Cambridge University Press, Cambridge, USA, 2004.

Connerney, J.E.P., M. H. Acuña, N.F. Ness, and T. Satoh, New models of Jupiter's magnetic field constrained by the Io flux tube footprint, J. Geophys. Res., 103, A6, 11929-11939, 1998.

Hospodarsky, G. B., Spaced-based search coil magnetometers, J. Geophys. Res., 121, 12068-12079, 2016.

Imai, M., K. Imai, C. A. Higgins, and J. R. Thieman, Angular beaming model of Jupiter's decametric radio emissions based on Cassini RPWS data analysis, Geophys. Res. Lett., 35, L17103, 2008.

Imai, M., K. Imai, C. A. Higgins, and J. R. Thieman, Comparison between Cassini and Voyager observations of Jupiter's decametric and hectometric radio emissions, $J$. Geophys. Res., 116, A12233, 2011.

Imai, M., A. Lecacheux, M. Moncuquet, F. Bagenal, C. A. Higgins, K. Imai, and J. R. Thieman, Modeling Jovian hectometric attenuation lanes during the Cassini flyby of Jupiter, J. Geophys. Res., 120, 1888-1907, 2015.

Imai, M., A. Lecacheux, T.E. Clarke, C. A. Higgins, M. Panchenko, J. Dowell, K. Imai, A. I. Brazhenko, A. V. Frantsuzenko, and A. A. Konovalenko, The beaming structures of Jupiter's decametric common S-bursts observed from the LWA1, NDA, and URAN2 radio telescopes, Astrophys. J., 826, id.176, 2016.

Imai, M., W. S. Kurth, G. B. Hospodarsky, S. J. Bolton, J. E. P. Connerney, and S. M. Levin, Direction-finding measurements of Jovian low-frequency radio components by Juno near Perijove 1, Geophys. Res. Lett., 44, 6508-6516, doi:10.1002/2017GL072850, $2017 \mathrm{a}$.

Imai, M., W.S. Kurth, G. B. Hospodarsky, S. J. Bolton, J. E. P. Connerney, S. M. Levin, A. Lecacheux, L. Lamy, and P. Zarka, Latitudinal beaming of Jovian decametric radio emissions as viewed from Juno and the Nançay Decameter Array, Geophys. Res. Lett., 44, 4455-4462, doi:10.1002/2016GL072454, 2017b.

Imai, M., W. S. Kurth, G. B. Hospodarsky, S. J. Bolton, J. E. P. Connerney, and S. M. Levin, Statistical study of latitudinal beaming of Jupiter's decametric radio emissions using Juno, Geophys. Res. Lett., 44, 4584-4590, doi:10.1002/2017GL073148, 2017c.

Kurth, W. S., G. B. Hospodarsky, D. L. Kirchner, B. T. Mokrzycki, T. F. Averkamp, W. T. Robison, C. W. Piker, M. Sampl, and P. Zarka, The Juno Waves investigation, Space Sci. Rev., 213, 347-392, doi:10.1007/s11214-017-0396-y, 2017a. 
Kurth, W. S., M. Imai, G. B. Hospodarsky, D. A. Gurnett, P. Louarn, P. Valek, F. Allegrini, J. E. P. Connerney, B. H. Mauk, S. J. Bolton, S. M. Levin, A. Adriani, F. Bagenal, G. R. Gladstone, D. J. McComas, and P. Zarka, A new view of Jupiter's auroral radio spectrum, Geophys. Res. Lett., 44, 7114-7121, doi:10.1002/2017GL072889, $2017 \mathrm{~b}$.

Kurth, W.S., M. Imai, G. B. Hospodarsky, D. A. Gurnett, S. S. Tetrick, S.-Y. Ye, S. J. Bolton, J. E. P. Connerney, and S. M. Levin, First observations near Jupiter by the Juno Waves investigation, in Planetary Radio Emissions VIII, edited by G. Fischer, G. Mann, M. Panchenko, and P. Zarka, Austrian Academy of Sciences Press, Vienna, $1-12,2017 \mathrm{c}$.

Ladreiter, H. P., P. Zarka, and A. Lecacheux, Direction finding study of Jovian hectometric and broadband kilometric radio emissions: Evidence for their auroral origin, Planet. Space Sci., 42, 913-931, 1994.

Lamy, L., P. Zarka, B. Cecconi, L. Klein, S. Masson, L. Denis, A. Coffre, and C. Viou, 1977-2017: 40 years of decametric observations of Jupiter and the Sun with the Nançay Decameter Array, in Planetary Radio Emissions VIII, edited by G. Fischer, G. Mann, M. Panchenko, and P. Zarka, Austrian Academy of Sciences Press, Vienna, 455-466, 2017.

Leblanc, Y., On the arc structure of the DAM Jupiter emission, J. Geophys. Res., 86, 8546-8560, 1981.

Lecacheux, A., Direction finding of a radiosource of unknown polarization with short electric antennas on a spacecraft, Astron. Astrophys., 70, 701-706, 1978.

Lecacheux, A., The Nançay Decameter Array: A useful step towards giant, new generation radio telescopes for long wavelength radio astronomy, in Radio Astronomy at Long Wavelengths, Geophys. Monogr., 119, p.321, 2000.

Louarn, P., F. Allegrini, D. J. McComas, P. W. Valek, W. S. Kurth, N. André, F. Bagenal, S. Bolton, J. Connerney, R. W. Ebert, M. Imai, S. Levin, J. R. Szalay, S. Weidner, R. J. Wilson, and J. L. Zink, Generation of the Jovian hectometric radiation: First lessons from Juno, Geophys. Res. Lett., 44, 4439-4446, doi:10.1002/2017GL072923, 2017.

Sampl, M., W. Macher, T. Oswald, D. Plettemeier, H. O. Rucker, and W. S. Kurth, Juno model rheometry and simulation, Radio Sci., 51, 1627-1635, doi:10.1002/2016RS005954, 2016.

Scarf, F. L. and D.A. Gurnett, A plasma waves investigation for the Voyager mission, Space Sci. Rev., 21, 289-308, 1977.

Treumann, R.A., The electron-cyclotron maser for astrophysical application, Astron. Astrophys. Rev., 13, 229-315, 2006.

Warwick, J. W., J. B. Pearce, R. G. Peltzer, and A. C. Riddle, Planetary Radio Astronomy experiment for Voyager missions, Space Sci. Rev., 21, 309-327, 1977.

Zarka, P., Auroral radio emissions at the outer planets: Observations and theories, J. Geophys. Res., 103, 20159-20194, 1998. 
